\title{
Effect of cross-type bias in a two-dimensional array of short Josephson junctions
}

Filatrella, G.; Pedersen, Niels Falsig; Wiesenfeld, K.

\section{Published in:}

Applied Physics Letters

Link to article, DOI:

10.1063/1.120938

Publication date:

1998

Document Version

Publisher's PDF, also known as Version of record

Link back to DTU Orbit

Citation (APA):

Filatrella, G., Pedersen, N. F., \& Wiesenfeld, K. (1998). Effect of cross-type bias in a two-dimensional array of short Josephson junctions. Applied Physics Letters, 72(9), 1107-1109. https://doi.org/10.1063/1.120938

\section{General rights}

Copyright and moral rights for the publications made accessible in the public portal are retained by the authors and/or other copyright owners and it is a condition of accessing publications that users recognise and abide by the legal requirements associated with these rights.

- Users may download and print one copy of any publication from the public portal for the purpose of private study or research.

- You may not further distribute the material or use it for any profit-making activity or commercial gain

- You may freely distribute the URL identifying the publication in the public portal

If you believe that this document breaches copyright please contact us providing details, and we will remove access to the work immediately and investigate your claim 


\title{
Effect of cross-type bias in a two-dimensional array of short Josephson junctions
}

\author{
G. Filatrella $a^{a)}$ \\ Department of Physics and INFM UNIT, University of Salerno, I-84081 Baronissi (SA), Italy \\ N. F. Pedersen \\ Physics Department, The Technical University of Denmark, DK-2800 Lyngby, Denmark \\ K. Wiesenfeld \\ School of Physics, Georgia Institute of Technology, Atlanta, Georgia 30332
}

(Received 28 August 1997; accepted for publication 23 December 1997)

We investigate numerically the effect of cross-type bias on two-dimensional arrays of short Josephson junctions. We have demonstrated that, for the simplest circuit, this type of bias is able to phase lock the junctions yielding a substantial improvement over ordinary biasing schemes. (C) 1998 American Institute of Physics. [S0003-6951(98)00509-9]

A Josephson junction oscillator for the millimeter and submillimeter band is one of the most promising applications of superconducting electronics. It would provide an excellent source (tunable frequency, narrow linewidth) in a region of the spectrum not easily accessible to traditional semiconductor technology.

The most serious drawback for such applications is the small power emitted by a single Josephson junction, and therefore efforts have been made to build series arrays of coherently working junctions to circumvent this difficulty. Unfortunately, the phase lock of a large number of Josephson junctions has posed a serious challenge for experimentalists, in spite of some success. ${ }^{1}$ A starting point to explain the difficulties is the observation that, in a lump circuit description, unloaded series arrays do not offer any coupling mechanism. To detect radiation an external load is needed, and this load will surely provide a coupling mechanism. However, the mere presence of an external load does not guarantee stable locking. One difficulty has been shown to arise from a surprising property of series arrays of Josephson junctions, i.e., the appearance of Lyapunov exponents that are exactly zero. ${ }^{2}$ It has further been recognized that loaded Josephson series arrays can have very unusual phase space dynamics due to the underlying structure of the governing dynamical equations: Watanabe and Strogatz ${ }^{3-5}$ have shown that there are $N-3$ constants of motion ( $N$ being the number of junctions), which implies very weak locking tendencies.

To circumvent the difficulties intrinsic to series arrays it has been proposed to use instead two-dimensional arrays, with the hope that the extra constraints due to the fluxoid quantization in the superconducting loop would offer an intrinsic (i.e., not due to the external load) coupling mechanism. Indeed experiments have demonstrated that emission from two-dimensional ${ }^{6,7}$ and from one-dimensional series array ${ }^{8}$ may be useful for practical applications. Simulations have also shown ${ }^{9}$ that parallel arrays are somewhat more stable against the spread of the fabrication parameters than series arrays. Analytic and numerical analysis have even

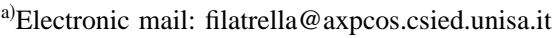

hinted that in some range of the parameters disorder can enhance the coupling. ${ }^{10,11}$

On the other hand, it has been demonstrated, ${ }^{12}$ when neglecting inductance effects, that the in-phase solution of two-dimensional arrays suffers from some of the same problems as those found in phase locking of series arrays. The key point of this problem is that when each row of the twodimensional array is oscillating in phase (i.e., all the vertical junctions in a row are phase locked), the horizontal junctions are inactive and there is no coupling mechanism between rows.

To couple together different rows various mechanisms have been proposed. The earliest is probably the use of triangular arrays. Numerical simulations ${ }^{13}$ have demonstrated that three junctions in a triangular loop may phase lock and that the system is stable for a large range of parameters. It has also been proposed ${ }^{14}$ to apply an external magnetic field to break the perfect symmetry of the in-phase mode across a row. The drawback of this method is that the frequency locked junctions do not oscillate in phase yet another proposal is to introduce more junctions in each loop. ${ }^{15}$

The purpose of this letter is to propose a way to overcome the decoupling between rows in a new way, namely by introducing a nontraditional current bias scheme. This will result in a more effective phase lock between the junctions and therefore in a circuit with better performances as a microwave generator. Specifically, we will show that a crosstype bias can be effective in driving the system into a stable frequency locked state without the need of an external load. This idea is similar to the one investigated by Larsen and Benz using a somewhat simpler $\left(2\right.$ cell) architecture. ${ }^{16}$

Figure 1 (a) shows a standard $2 \times 2$ cell array with a total of 12 junctions. The fundamental idea behind the present work is to remove the row switching and neutral stability by introducing a gradient in the row direction. We achieve this by using a uniform bias current but applying it at the top and removing it at the right hand side as shown in Fig. 1(a). The corresponding set of ordinary differential equations of the circuit have been numerically integrated using a fourth-order Runge-Kutta algorithm. 


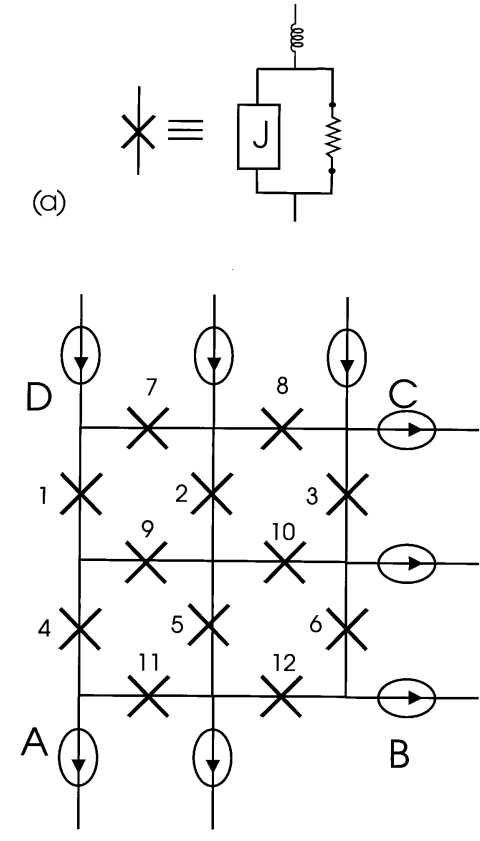

(b)

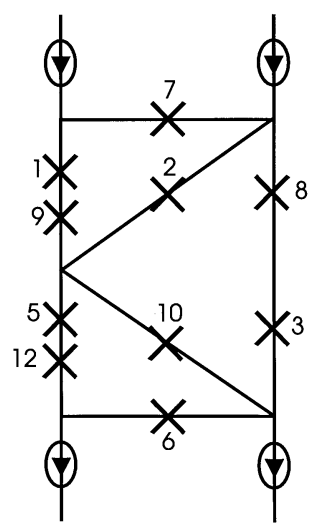

(c)

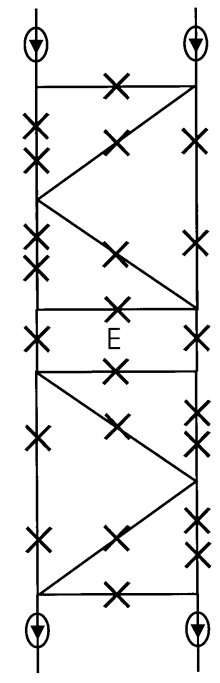

FIG. 1. Schematic circuit model for a two-dimensional array with cross-type bias. (a) The array; (b) an approximate equivalent circuit for the case with cross-type bias and negligible voltage across junctions 4 and 11 in (a); (c) a series connection of two circuits of the type in (b).

Each of the junctions are characterized by a critical current $I_{c}$ and a normal state resistance $R$, while the McCumber parameter $\beta_{c}$ is assumed to be zero. The loop inductance is $L$ and its value is specified by the parameter $\beta_{L}=2 \pi L I_{c} / \Phi_{0}$. The bias current $\gamma$ is normalized to the single junction critical current $I_{c}$. The unit of time is $\Phi_{0} / R I_{c}$.

To write the equations in a convenient and compact form we first introduce the auxiliary variables $I_{i}^{s}$, defined as the screening currents in the $i$ th loop. These currents are determined by the fluxoid condition

$$
n \Phi_{0}=\Phi^{\text {total }}=L I^{s}+\Phi^{\text {external }}+\sum_{\text {loop }} \phi
$$

where $\phi_{i}$ denotes the phase difference across the $i$ th junction. Solving Eq. (1) with respect to $I^{s}$ for each loop gives $I^{S}$ as a function of the phases $\phi_{i}$. The circuit equations are obtained using Kirchhoff's Laws to find the current in each Downloaded 15 Jan 2010 to 192.38.67.112. Redistribution subject

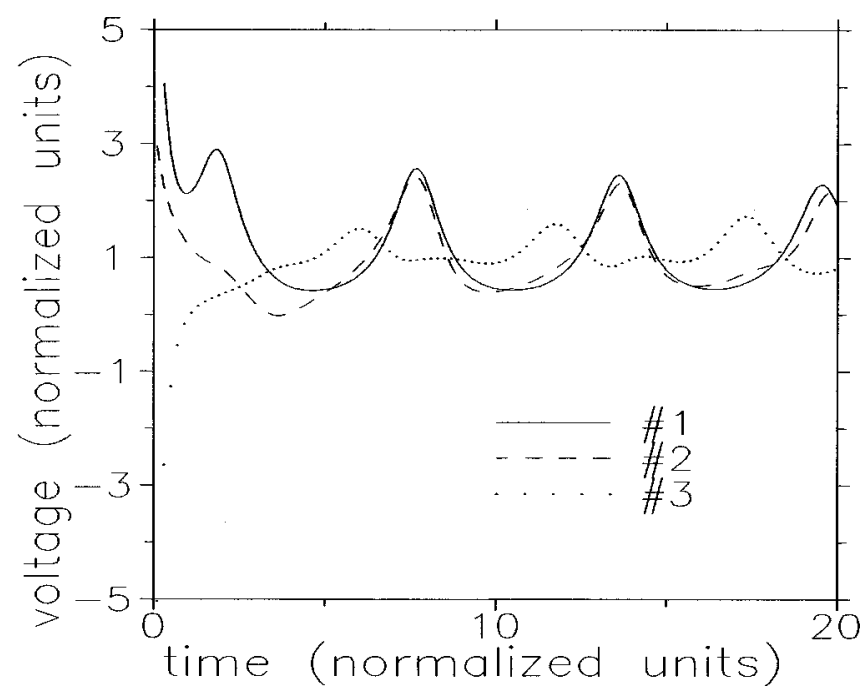

FIG. 2. $\dot{\phi}(t)$ for random initial conditions for the circuit in Fig. 1(b). Parameters of the simulations are: $\gamma=2, \beta_{L}=1$.

branch. For example, the equation of motion across junction No. 1 [see Fig. 1(a)] reads, in normalized units:

$$
\dot{\phi}_{1}=\sin \phi_{1}+\frac{1}{\beta_{L}}\left(-\phi_{1}-\phi_{7}+\phi_{2}+\phi_{9}\right)+\gamma .
$$

The biasing scheme we propose is to inject current in the nodes along the branch $\mathrm{D}-\mathrm{C}$ and extract it from the nodes belonging to the branch $\mathrm{C}-\mathrm{B}$. (In the traditional biasing scheme the current would be extracted along the nodes parallel to the A-B branch.)

We note that Eq. (1) is an approximation: ${ }^{17}$ the first term on the right-hand side is but the first term of the field generated by all the currents present in the circuit; ${ }^{18}$ a more complete approach should require the use of the full inductance matrix. We have checked that our results do not substantially change by introducing in addition one more term (next nearest neighbors) of the full expansion. For a discussion of the limits of the validity of the different approaches and of the physical role of the screening currents see Ref. 19.

Figure 2 shows that this biasing scheme successfully removes the neutral stability. The parameters are $\gamma=2$ and $\beta_{L}=1$. For initial conditions we tried several random sets of numbers. Figure 2 shows the results for one choice, and these results are typical. The system finds a steady state very quickly, and this state was found to be independent of the initial conditions, e.g., in the steady state the phase relation between the oscillations in the various junctions is always the same. The different curves shown in Fig. 2 show different voltages labeled according to the numbering in Fig. 1(a). Thus, the voltage $V_{3}$ corresponds to the voltage across junction No. 3, etc.

Figure 3 shows how the voltages are distributed across the different junctions. Note that we only show half of the voltages, because the system is (anti) symmetric with respect to a diagonal line from point $\mathrm{A}$ to point $\mathrm{C}$ in Fig. 1(a). As voltages are measured positive in the $X$ direction and $Y$ direction we find, for example, that $V_{4}=-V_{11}, V_{9}=-V_{5}$, etc. As can be seen from the figure, all the voltages oscillate with the same frequency. Note, too, that the currents (large voltages) have a tendency to go the shortest way (impedanceto AlP license or copyright; see http://apl.aip.org/apl/copyright.jsp 


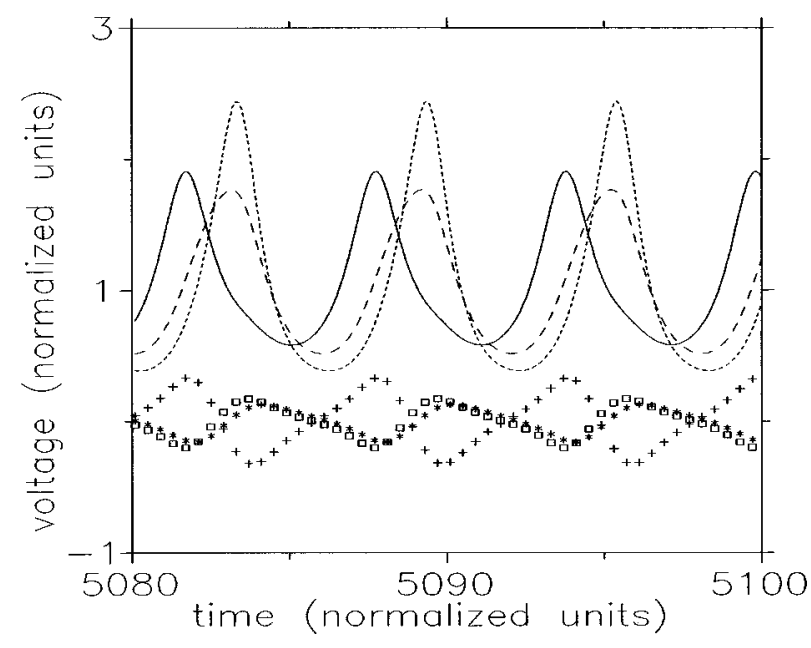

FIG. 3. $\dot{\phi}_{1}(t)$ of the $i$ th junction of the array in Fig. 1(b). Symbols refer to $i=1$ (solid line), 2 (larger dotted line), 3 (smaller dotted line), 4 (stars), 5 (squares), 6 (pluses). Parameters of the simulations are: $\gamma=2, \beta_{L}=1$.

wise) from bias input to bias output (i.e., for example the paths 2 and 10) whereas the paths 4 and 11 only has zero average voltage and little ac voltage. All the paths transverse to the main current flow (i.e., 7, 9, 5, 6) are activated at a low level with nearly zero average; nevertheless, the coupling is sufficient to lock the junctions and prevent neutral stability. If desired, a load can now be connected from B to D by which the voltages across most of the circuit add in an almost a series configuration. Since the voltages across 4 and 11 are small, Fig. 1(a) suggests that we may remove those junctions without disturbing the overall behavior too much, and in so doing make the topological transformation of the regular square array in Fig. 1(a) to the irregular ladder array in Fig. 1(b). We have confirmed by simulation that junctions 4 and 11 may be removed without changing the overall behavior too much.

In order to test whether our biasing scheme remains successful for larger arrays, we also ran simulations for a $3 \times 3$ cell circuit. Interestingly, we found that the diagonally symmetric biasing scheme indicated in Fig. 1(a) was not suffi-

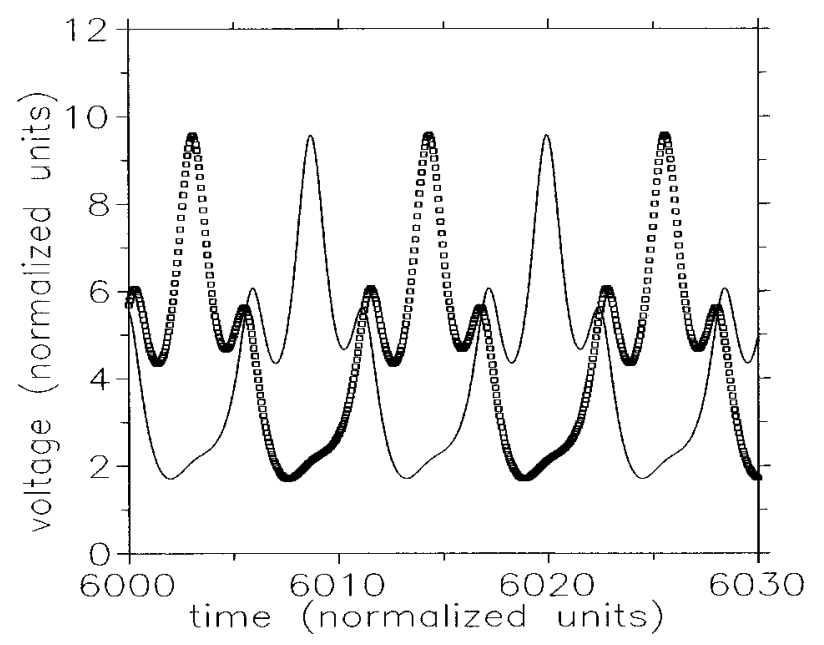

FIG. 4. $\dot{\phi}(t)$ of the junctions in the array depicted in Figs. 1(c) and 1(b). Squares and the solid line refer to the total voltage drop on the left hand side and right hand side of the circuit in Fig. 1(c), respectively. Parameters of the

${ }^{19}$ A. Petraglia, G. Filatrella, and G. Rotoli, Phys. Rev. B 53, 2732 (1996)
Downloaded 15 Jan 2010 to 192.38.67.112. Redistribution subject to AlP license or copyright; see http://apl.aip.org/apl/copyright.jsp cient to induce stable frequency locking. Rather, it was necessary to introduce a gradient in the applied bias current itself in order to achieve single frequency behavior. On the other hand, we find that two circuits of the type depicted in Fig. 1(b) can be locked if biased in series.

We also performed simulations with ladder arrays of the type shown in Fig. 1(c). Figure 4 displays the total voltage across a series connection of the two ladders as displayed in Fig. 1(b). The two ladders are coupled through loop E in Fig. 1(c). In order to increase the coupling between the two ladders (for computational reasons) we have chosen the coupling circuit to have $\beta_{L}=0.1$.

The figure shows the total voltage at both the left- and the right-hand side. We clearly see there is complete frequency locking even in the presence of disorder. The total voltage across the two arrays becomes large and can be further increased by adding more units in series. We notice from Fig. 4 that the left- and right-hand side oscillate in antiphase. In other cases we found that the two sides oscillate in-phase. Notice also from the voltage waveform in Fig. 4, that there is a high harmonic content. This could be used to increase the frequency of the series block oscillator.

One of the authors (G.F.) wishes to thank the Physics Department of the Technical University of Denmark where part of this work was carried out.

${ }^{1}$ S. Han, B. Bi, W. Zhang, and J. E. Lukens, Appl. Phys. Lett. 64, 1424 (1994); K. Wan, A. K. Jain, and J. E. Lukens, ibid. 54, 1805 (1989).

${ }^{2}$ P. Hadley, M. R. Beasley, and K. Wiesenfeld, Phys. Rev. B 38, 8712 (1988).

${ }^{3}$ S. Watanabe and S. H. Strogatz, Phys. Rev. Lett. 70, 2391 (1993).

${ }^{4}$ S. Watanabe and S. H. Strogatz, Physica D 74, 197 (1994).

${ }^{5}$ S. Watanabe and J. W. Swift, J. Nonlinear Sci. 7, 503 (1997).

${ }^{6}$ S. P. Benz and C. J. Burroughs, Appl. Phys. Lett. 58, 2162 (1991).

${ }^{7}$ P. A. A. Booi and S. P. Benz, Appl. Phys. Lett. 64, 2163 (1994).

${ }^{8}$ P. A. A. Booi and S. P. Benz, Appl. Phys. Lett. 68, 3799 (1996).

${ }^{9}$ R. L. Kautz, IEEE Trans. Appl. Supercond. 5, 2702 (1995).

${ }^{10}$ Y. Braiman, W. L. Ditto, K. Wiesenfeld, and M. L. Spano, Phys. Lett. A 206, 54 (1995).

${ }^{11}$ A. S. Landesberg, Y. Braiman, and K. Wiesenfeld, Phys. Rev. B 52, 15459 (1995).

${ }^{12}$ K. Wiesenfeld, S. P. Benz, and P. A. A. Booi, J. Appl. Phys. 76, 3835 (1994).

${ }^{13}$ S. P. Yukon and N. C. H. Lin, in Nonlinear Superconducting Devices and High-T $T_{c}$ Materials, edited by R. D. Parmentier and N. F. Pedersen (World Scientific, Singapore, 1995), p. 137; S. P. Yukon and N. C. H. Lin, in Superconducting Devices and High- $T_{c}$ Materials, edited by C. Giovannella and M. Tinkham (World Scientific, Singapore, 1995), p. 351.

${ }^{14}$ G. Filatrella and K. Wiesenfeld, J. Appl. Phys. 78, 1878 (1995); Nonlinear Superconducting Devices and High- $T_{c}$ Materials, edited by R. D. Parmentier and N. F. Pedersen (World Scientific, Singapore, 1995), p. 329.

${ }^{15}$ M. Darula, A. Darulova, P. Seidel, S. Benacka, and B. Misanik, Nonlinear Superconducting Devices and High-T ${ }_{c}$ Materials, edited by R. D. Parmentier and N. F. Pedersen (World Scientific, Singapore, 1995), p. 201; M. Darula, P. Seidel, F. Busse, and S. Benacka, J. Appl. Phys. 74, 2674 (1993).

${ }^{16}$ B. H. Larsen and S. P. Benz, Appl. Phys. Lett. 66, 3209 (1995).

${ }^{17}$ K. Nakajima and Y. Sawada, J. Appl. Phys. 52, 5732 (1981).

${ }^{18}$ J. R. Phillips, H. S. J. van der Zant, J. White, and T. P. Orlando, Phys. Rev. B 47, 5219 (1993).

${ }^{19}$ A. Petraglia, G. Filatrella, and G. Rotoli, Phys. Rev. B 53, 2732 (1996) AIP license or copyright; see http://apl.aip.org/apl/copyright.jsp 